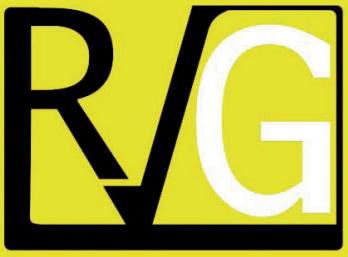

Julio - Septiembre, 2021

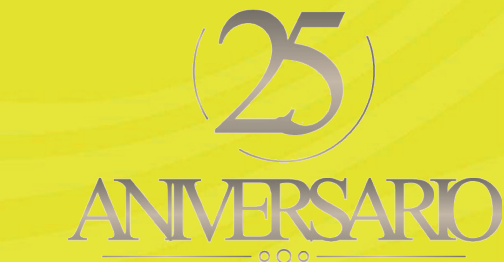

ANMERSARO
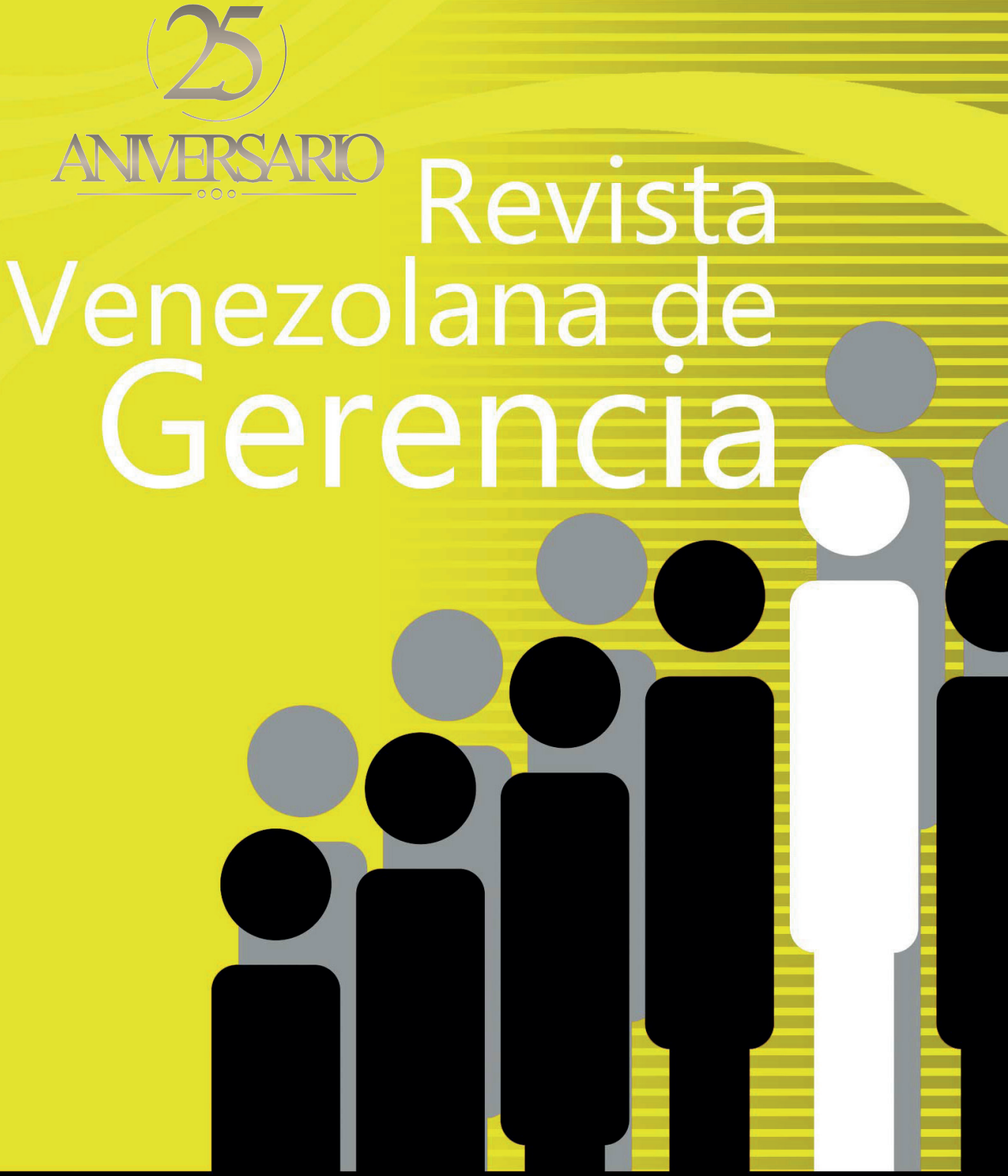

UNIVERSIDAD DEL ZULIA (LUZ)

Facultad de Ciencias Económicas y Sociales Centro de Estudios de la Empresa

ISSN 1315-99

Esta obra está bajo una licencia de Creative Comm Reconocimiento-NoComercial-Compartirlgual 3.0 Unpo http://creativecommons.org/licenses/by-nc-sa/3.0/deed.es 
COMO CITAR: Moreno, C., Johnson, V., y Puyuelo, M. (2021). Realidades de Emprendimiento en Pequeñas y Medianas Empresas en Chile: uso del diseño. Revista Venezolana de Gerencia (RVG), 26(95), 898-917. https://doi. org/10.52080/rvgluz.27.95.28
Universidad del Zulia (LUZ)

Revista Venezolana de Gerencia (RVG)

Año 26 No. 95 Julio-Septiembre 2021, 898-917

ISSN 1315-9984 / e-ISSN 2477-9423

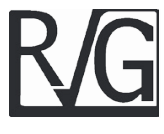

\title{
Realidades de Emprendimiento en Pequeñas y Medianas Empresas en Chile: uso del diseño
}

\author{
Moreno Muñoz, Cristóbal* \\ Johnson Vera, Vader** \\ Puyuelo Cazorla, Marina***
}

\section{Resumen}

El siguiente artículo presenta un estudio multimodal, cuantitativo y cualitativo, para comparar diversas realidades socioeconómicas chilenas con el objetivo de identificar los factores sociales que afectan el avance económico de las pequeñas y medianas empresas (PYMEs) y coartan el uso estratégico del Diseño en los emprendimientos. Para realizar este análisis, se tomará como objeto de estudio a tres Centros de Desarrollo de Negocios (CDN) de la Región Metropolitana de Chile, según la comuna en la cual se ubican y el ranking de esta en el Índice de Prioridad Social (IPS), lo cual determina su nivel socioeconómico (bajo, medio y alto). Esto se complementará con entrevistas a los expertos de estos CDN para realizar una caracterización de los emprendimientos en estas comunas. Las realidades estudiadas denotan que existe una correlación positiva entre nivel socioeconómico y el grado de sofisticación de la propuesta del CDN, de

\section{Recibido: $12.01 .21 \quad$ Aceptado: 10.04.21}

* Diseñador Industrial de la Universidad Tecnológica Metropolitana de Chile. Master en Pedagogía de la Universidad Mayor de Chile. Doctorando en la Universitat Politécnica de Valencia. Académico Asociado, Jefe de Carrera Diseño Industrial de la Facultad Tecnológica de la Universidad de Santiago de Chile. cristobal. moreno.m@usach.cl, ORCID: https://orcid.org/0000-0002-6477-5118

** Ingeniero Civil Eléctrico de la Universidad de Chile. MSc in Innovation, Entrepreneurship and Management, University of Nottingham, UK. CEO de ICInnovations. Profesor Adjunto I de la Facultad Tecnológica de la Universidad de Santiago de Chile. vader.johnson@usach.cl, ORCID: https://orcid.org/0000-0002-5268-4171

*** Licenciada en Bellas Artes en la Universitat Politécnica de Valencia, Doctora en la Escuela Superior de Arquitectura Universitat Politécnica de Valencia. Profesora Titular en la Escuela Superior de Arquitectura Universitat Politécnica de Valencia. mapuca@ega.upv.es,ORCID: https://orcid.org/0000-0002-7720-5907 
su planteamiento estratégico y la inserción del Diseño en sus procesos de negocios. Existe una oportunidad en el contexto de las PYMEs para introducir la disciplina del diseño como una herramienta estratégica que permita fortalecer el ecosistema de emprendimiento, dándoles a los emprendedores instrumentos que les permitan sofisticar sus negocios.

Palabras clave: Emprendimiento; Diseño; PYME; Centros de Desarrollo de Negocios; Chile.

\title{
Entrepreneurship Realities in Small and Medium Enterprises in Chile: use of design
}

\begin{abstract}
The following article presents a multimodal study, quantitative and qualitative, to compare diverse Chilean socioeconomic realities with the objective of identifying the social factors that affect the economic advance of small and medium businesses (SMBs) and limit the strategic use of Design in entrepreneurship. To carry out this analysis, the object of this study will be three Centers of Business Development (CBD) in the Metropolitan Region o Santiago, in Chile, according to the commune in which they are located and its ranking in the Index of Social Priority, which determines its socioeconomic level (low, medium and high). This will be complemented with interviews with experts of the CDB to compose a characterization of entrepreneurship in those communes. The studied realities denote a positive correlation between socioeconomic level and the degree of sophistication of CBD proposal, its strategic guidelines and the insertion of Design in their business processes.
\end{abstract}

Key Words: Entrepreneurship; Design; SMBs; Centers of Business Development; Chile.

\section{Introducción}

Según cifras del Ministerio de Economía, Fomento y Desarrollo de Chile, hasta el año 2018, el 52,1\% de la fuerza laboral trabajaba en pequeñas y medianas empresas (PYMEs), es decir, empresas cuyos ingresos anuales oscilan aproximadamente entre 100.000 y 4.000 .000 USD (Ministerio de
Economía, Fomento y Turismo, 2014). La naturaleza de estas empresas varía; sin embargo, como indican Puente, Espitia \& Cervilla (2019), la necesidad es un factor recurrente en su surgimiento, especialmente en América Latina, constituyéndose como una alternativa forzosa al trabajo tradicional dado por un empleador. Este apremio conlleva una falta de planificación y uso estratégico 
de herramientas como el Diseño, que podría mejorar el producto o servicio ofrecido; la forma en la cual se presenta; el propósito que cumple; y la manera en que llega al usuario.

En este contexto, este estudio plantea la disciplina del Diseño como una herramienta estratégica altamente subvalorada, que es capaz de aportar no solo un valor agregado a los productos y servicios de una empresa sino también a los procesos productivos y comerciales (Moreno, Aguirre y Puyuelo, 2019). Para efectos de esta investigación, el Diseño a modo de disciplina considera todas las competencias que conducen a una visión global orientada hacia la planificación estratégica de un producto final, configurándose más allá de los alcances de profesiones como el Diseño Gráfico, el Diseño Industrial, el Diseño de servicios, entre otros. El diseño como disciplina comprende la propuesta de soluciones a problemas concretos, desde la producción de un artefacto hasta como la concepción de un proceso estratégico (Consejo nacional de la Cultura y las Artes, 2017:11). Por esto, la disciplina tiene «el potencial de fomentar el crecimiento económico, la creación de empleos y ganancias de exportación» (Fonseca, 2008), y se establece como factor crucial del intercambio económico y cultural que trasciende la representación formal de los objetos y los procesos (Gamonal, 2011).

En sintonía con ello, Adriasola (2019) indica que el Diseño en los negocios se debe visualizar como una disciplina centrada en las personas y ejercida desde perspectivas como el Design Thinking (Leinonen \& Gazulla, 2014; Ortega y Ceballos, 2015) y el modelo de Doble Diamante (Carayannis \& Wang, 2012). Estos modelos de trabajo implican aceptar que los errores son una experiencia del aprendizaje, parte de un proceso innovador, y "subproductos inevitables de un proceso de experimentación" (BID, 2006, en Adriasola, 2019). El mismo estudio apunta a que, en las economías desarrolladas, tomar riesgos e innovar a través del error es una práctica común. Empero, en países en vías de desarrollo, estos enfoques no son fáciles de imitar, pues lo procesos de experimentación implican estar dispuesto a asumir pérdidas en caso de error, algo difícil de hacer en contextos de recursos limitados que obstaculizan el recuperarse, conduciendo al fracaso. Sin embargo, este factor limitante no es el único que determina la baja incorporación del Diseño en los negocios. Aún sin grandes problemas financieros, muchas PYMEs no encuentran la forma de encauzar sus recursos hacia las inversiones en innovación, infraestructura y desarrollo sostenible que podrían impulsar su negocio hacia el éxito y sustentabilidad económica, por lo que ese precepto requiere de cambios sustanciales y multifactoriales que garantice una nueva proyección innovadora (Maldonado et al. 2021).

Según Nordfors, Cerf y Senges (2016), otros factores sociales que pueden incidir en esto son la educación de sus fundadores, su capital cultural, su contexto social y el acceso a cierto lenguaje compartido necesario para la generación de nuevas ideas, incluso cuando el capital monetario disponible no es una limitante.

Bajo esta mirada, el presente artículo busca determinar factores sociales que influyen en el avance económico de las PYMEs, comparando diversas realidades socioeconómicas del emprendimiento chileno e identificando su nivel de sofisticación 
en el uso estratégico del Diseño como herramienta de negocios. Para realizar este análisis, este articulo inicia con una breve contextualización de la función del Diseño en las empresas como herramienta estratégica para el avance económico. Luego caracteriza a los Centros de Desarrollo de Negocios (CDN), instituciones que apoyan PYMES a lo largo de Chile. Tras ello, se define el Índice de Prioridad Social (IPS), indicador de desarrollo socioeconómico usado por el Gobierno de Chile para focalizar sus políticas públicas (Ministerio de Desarrollo Social y Familia, 2019), el que es posteriormente utilizado para seleccionar CDNs representativos de tres diferentes niveles socioeconómicos (bajo, medio y alto). En base a este análisis, se realizan a entrevistas a expertos de los CDN para pesquisar aspectos claves de la realidad socioeconómica de sus emprendimientos como el Perfil del emprendedor; las Urgencias del emprendedor; La función del Diseño; y los Ecosistemas e Innovación. Finalmente, se presentan los hallazgos sobre cómo el capital económico y cultural influyen en el nivel de sofisticación del uso del Diseño para el avance económico de las PYMEs.

\section{Diseño como herramienta estratégica del emprendimiento}

Para instalar el diseño en la cultura empresarial como una herramienta estratégica, se debe entender, primeramente, como un agente articulador entre el área de marketing y el área de ingeniería. Ello implica que el Diseño puede crear puentes entre el desarrollo comercial y la generación de nuevos mercados y necesidades, hasta la gestión de este desarrollo, su producción y su optimización técnica con la intención de generar mejores ingresos, encontrar aperturas a nuevos mercados, disminuir costos, mejorar la identidad y el reconocimiento de marca y la calidad del producto, entre otros factores (Moreno y Jeno, 2018).

Con estos propósitos en mente, se puede visualizar el escalamiento y la inserción del Diseño en las empresas (Ramlau, 2004). El modelo del escalamiento e inserción, inicialmente planeado por Viladás (2010) propone diversos niveles, en orden ascendente, en los cuales se ubica una empresa dependiendo de su uso del Diseño como herramienta estratégica. Este modelo se apoya en la Escalera del Diseño (2003), un marco referencial de Dinamarca que se usa como un medio gráfico para explicarles a las empresas que Diseño puede tener una función mucho más compleja y profunda que estilo o estética, al igual que una medida para tasar los beneficios económicos de las empresas a nivel nacional (Fleetwood, 2005:4).

La figura 1 representa los cuatros escalones de base de posicionamiento de Diseño, denotando que, a mayor uso del Diseño en las empresas, más elevado y sofisticado es el lineamiento estratégico de los planes de negocios. Esto implica que, las empresas confían en el diseño para el desarrollo de nuevos productos $\mathrm{y}$, además, permiten que el diseño forme parte de la estrategia corporativa. Poseen una fuerte identidad de marca que las diferencias de otras empresas ya que no tienen necesidad de competir por precio o calidad, dado que estos elementos están garantizados en una filosofía de la marca que ha sido diseñada para dar confianza que se proyecta hacia el mercado. Asimismo, Solis (2021) señala que los 
esfuerzo organizacionales han de estar orientados hacia la transformación social, económica y cultural de la sociedad como fuente generadora de productividad

Por sobre el último escalón, Viladás (2010) plantea una nueva concepción orientado a la innovación y gestión, en los cuales se apunta hacia un cambio fundamental en los empresarios que les requiere tener mentalidad de diseñadores, implicando mayor apertura a riesgos. Esta nueva figura 1 de empresario está siempre enfocada hacia el usuario, hace pruebas y preguntas intuitivas y es capaz de adoptar nuevos conceptos según los cambios en su entorno.

\section{Figura 1}

\section{La escalera del Diseño: Escalamiento e inserción del Diseño en empresas}

\section{Escalamiento e Inserción del Diseño}

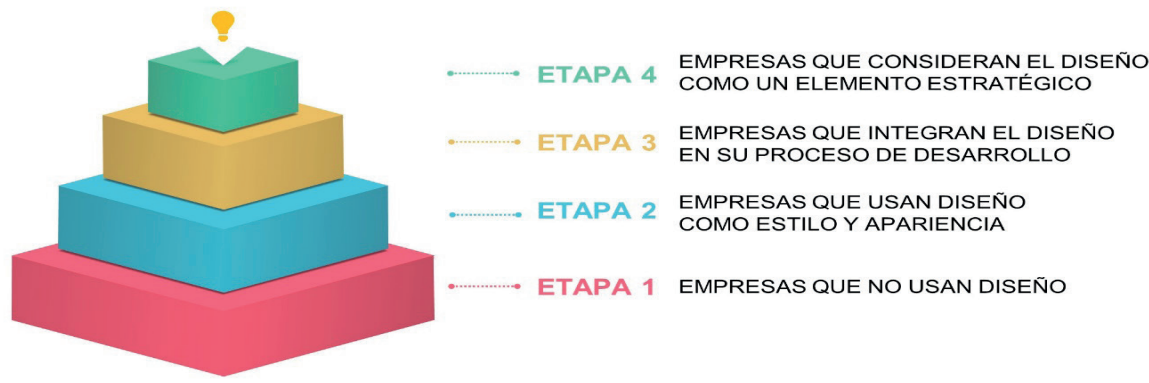

Fuente: Figura adaptada de Viladás, 2010.

Estas nociones están apoyadas por planteamientos de expertos como Lawlor et al. (2015), quien adjudica el impacto económico del Diseño en base a factores adicionales como la capacidad estratégica para responder a los desafíos de la actualidad y a las necesidades de las personas en la forma correcta, al tiempo correcto; la creación de valor basado en intangibles y en el aumento de la masa de los derechos de autor y propiedad industrial; la creación de productos distintivos y atractivos acompañados de un precio pagado mayor que su producción; y, por último, el ahorro de costos generados a través de los medios del Diseño.

\section{Centros de Desarrollo de Negocios (CDN)}

La red de CDN nace en junio, 2014, con el fin de promocionar el emprendimiento y crecimiento de las 
PYMEs. Surge desde el Servicio de Cooperación Técnica [SERCOTEC] (SERCOTEC, 2019) a partir de un acuerdo suscrito entre los gobiernos de Chile y Estados Unidos, con el apoyo técnico de la organización Small Business Development Centers (SBDC) (America's Small Business Development Centers, 2020). Este acuerdo tiene el objetivo de poner a disposición de los y las emprendedoras de las PYMEs servicios de apoyo para asegurar el impacto económico, con foco el aumento de la productividad y sostenibilidad de las empresas.

En Chile, los CDN atienden a más de 300 PYMEs que tratan en dos grupos específicos de clientes: emprendedores que están recién empezando a formalizarse y empresas, que ya están formalizadas. A ambos tipos, se les brinda apoyo especializado de mentores expertos en las diferentes áreas del negocio, de manera individual y gratuitamente, con el objetivo de fortalecer sus capacidades y desarrollar de manera óptima las actividades de la empresa, de manera sustentable e inclusiva. Además, los Centros ofrecen una asesoría especial, dirigida a mujeres, jóvenes y personas con discapacidad con vocación emprendedora, que les permite adoptar una metodología de enseñanza universal. Cada CDN atiende alrededor de cien PYMEs en un determinado momento; sin embargo, esta cifra puede aumentar o disminuir dependiendo de la rotación de sus clientes.

\section{Caracterización Sociocultural: El Índice de Prioridad Social (IPS)}

El Índice de Prioridad Social es un instrumento de medición del nivel de desarrollo socioeconómico de cada comuna de la región relativo a la población que habita en ella. Este tipo de instrumento funciona como apoyo para las políticas públicas en la elaboración y desarrollo de programas destinados a mejorar aquellas áreas y poblaciones que presentan las mayores carencias relativas. En particular, este instrumento "es un indicador compuesto que integra aspectos relevantes del desarrollo social comunal, esto es, las dimensiones de: ingresos, educación y salud" (Ministerio de Desarrollo Social y Familia, 2019). Además, el índice funciona como ranking, ubicando a cada comuna en un lugar relativo a las 52 comunas totales de la Región Metropolitana de Chile. En este contexto, el IPS se constituye como:

Una investigación empírica que estudia un fenómeno contemporáneo dentro de su contexto de la vida real, especialmente cuando los límites entre el fenómeno y su contexto no son claramente evidentes. (...) Se basa en múltiples fuentes de evidencia, con datos que deben converger en un estilo de triangulación; $y$, también como resultado, se beneficia del desarrollo previo de proposiciones teóricas que guían la recolección y el análisis de datos (Yin, 1994 en Yacuzzi 2005).

Los indicadores de ingresos, educación y salud del IPS se miden en una escala normalizada de 0 a 100 . En esta concepción, 100 es el valor que representa la mayor prioridad, entendida como la peor situación, y 0 es la menor prioridad o prioridad nula, entendida como la mejor situación.

Para efectos de este estudio se considerarán exclusivamente las dimensiones de ingresos económicos y de educación, al ser estas las de mayor impacto directo en la actividad de los emprendimientos. Estas se definen a continuación: 


\subsection{Dimensión de Ingresos económicos:}

- Porcentaje de población comunal perteneciente al $40 \%$ de menores ingresos de la Clasificación Socio Económica (CSE): Se determina en base a los ingresos de los integrantes del hogar $y$ otros aspectos, como correcciones por un índice de necesidades, como reordenamiento en base a bienes y servicios a los que accede el hogar y que puedan inferir su nivel socioeconómico. Estos datos corresponden a la información recopilada por el Registro Social de Hogares (RSH) del Ministerio de Desarrollo Social de Chile.

- Ingreso promedio imponible de los afiliados vigentes al Seguro de Cesantía: Determina el potencial relativo de generación de ingresos del trabajo de la población ocupada, de una comuna en particular. Estos datos corresponden a la información recopilada por la Administración de Fondos de Cesantía (AFC), perteneciente a los ingresos laborales de la población ocupada afiliada al AFC durante el año 2015.

- Dimensión de Educación:

- Resultados de Prueba de Selección Universitaria (PSU): Corresponde al puntaje promedio por comuna, obtenido en la PSU de aquellos estudiantes que rindieron esta prueba por primera vez durante el proceso correspondiente a la promoción escolar 2017. Este indicador se construye con el puntaje promedio ponderado por los estudiantes en las pruebas de Lenguaje y Matemáticas de la comuna de residencia. Este resultado condiciona en gran medida las posibilidades de acceso a la educación superior, lo cual, a su vez condiciona sus opciones de inserción laboral y generación de ingresos en el futuro. Estos datos fueron recopilados por el Departamento de Evaluación, Medición y Registro Educacional de la Universidad de Chile (DEMRE).

- Porcentaje de Reprobación en la Enseñanza Media: Corresponde al porcentaje de alumnos que presenta reprobación en algún nivel correspondiente a la enseñanza media (educación secundaria). Estos datos se construyen en base al promedio de alumnos reprobados por comuna durante el periodo de 2011 a 2015. Estos datos tienen como fuente al Ministerio de Educación.

\section{Caracterizando a los Emprendedores: Miradas de Expertos}

Respecto del estudio cualitativo de esta investigación, se hará uso de la entrevista por su "exploración en profundidad de la perspectiva de los actores sociales (...) indispensables para una exacta aprensión y comprensión de las conductas sociales" (Robertt y Lisdero, 2016), para dimensionar cómo se transmite el Diseño desde los CDN, representados por sus directores, hacia los emprendedores y empresarios de cada comuna. En este contexto, los entrevistadores fueron los investigadores de esta investigación y los entrevistados fueron los directores y asesores de los CDN de las comunas de Estación Central, La Florida y Ñuñoa, a modo de expertos por su conocimiento y experiencia. 
Las entrevistas se realizaron en base a una pauta de preguntas abiertas sobre los emprendedores de PYMEs de cada centro y su uso del diseño, que se llevó a cabo durante los meses de septiembre y octubre del año 2020, mediante una videoconferencia por la plataforma Zoom que fue grabada $y$ transcrita posteriormente. A partir de la pauta de preguntas se pesquisaron cuatro categorías principales para caracterizar a cada centro, y los respectivos elementos que la configuran, cuadro 1.

\section{Cuadro 1 \\ Caracterización de los CDN}

\begin{tabular}{|c|c|c|c|}
\hline Categoría & Definición & Composic & ción \\
\hline Perfil del Emprendedor & $\begin{array}{l}\text { Esta categoría busca definir el tipo de } \\
\text { cliente (emprendedor o empresario) } \\
\text { que más atiende el Centro. }\end{array}$ & $\begin{array}{l}- \\
- \\
- \\
\text { servicios } \\
- \\
-\end{array}$ & $\begin{array}{l}\text { Edad } \\
\text { Nivel de estudios } \\
\text { Desarrollo de productos / } \\
\text { Necesidad de Innovación } \\
\text { Necesidad de Sustento }\end{array}$ \\
\hline $\begin{array}{l}\text { Urgencia del } \\
\text { Emprendedor }\end{array}$ & $\begin{array}{l}\text { Esta categoría identifica las } \\
\text { prioridades que el cliente busca } \\
\text { atender y cómo el Centro responde } \\
\text { a ello. }\end{array}$ & $\begin{array}{l}- \\
-\end{array}$ & $\begin{array}{l}\text { Contabilidad tributaria } \\
\text { Propuesta de Valor } \\
\text { Modelo de desarrollo Técnico }\end{array}$ \\
\hline La función del Diseño & $\begin{array}{l}\text { Esta categoría pretende establecer } \\
\text { la importancia que cada Centro le } \\
\text { atribuye al Diseño, cuáles son los } \\
\text { acercamientos y los intereses que } \\
\text { busca incidir en su cliente. }\end{array}$ & $\begin{array}{l}- \\
- \\
- \\
-\end{array}$ & $\begin{array}{l}\text { Comunicación digital } \\
\text { Materia gráfica } \\
\text { Valor del producto } \\
\text { Ferias / Exposiciones } \\
\text { Comercialización / Estrategia }\end{array}$ \\
\hline $\begin{array}{l}\text { Ecosistema e } \\
\text { Innovación }\end{array}$ & $\begin{array}{l}\text { Esta categoría indaga en la visión } \\
\text { de redes de apoyo e innovación que } \\
\text { mantiene el Centro. }\end{array}$ & $\begin{array}{l}- \\
- \\
\text { (academia } \\
- \\
-\end{array}$ & $\begin{array}{l}\text { Vinculación entre clientes } \\
\text { Redes con el entorno } \\
\text { gremios y empresas) } \\
\text { Interés por la Innovación } \\
\text { Presupuesto para Innovación } \\
\text { Cultura de Innovación }\end{array}$ \\
\hline
\end{tabular}

Fuente: Elaboración propia basados en las categorías, definiciones y elementos pesquisadas de entrevistas abiertas a expertos del CDN de Estación Central, La Florida y Ñuñoa.

\section{Nivel Socioeconómico del Emprendedor: Ingresos y Educación}

A continuación, se recopilan resultados sobre el nivel socio económico que establece el Índice de Prioridad Social de cada comuna estudiada. Estos resultados grafican que el nivel socio económico se configura en torno a tres factores: ingresos económicos, nivel de educación y acceso a salud. A mayor nivel socio económico de la comuna, menor será el ranking de IPS, ya que significa que un nivel socio económico alto, de grandes ingresos económicos, nivel mayor de educación y fácil acceso a la salud, implica que la comuna no tiene grandes prioridades sociales y el gobierno no tiene urgencia por mejorar 
Moreno Muñoz, Cristóbal; Johnson Vera, Vader; Puyuelo Cazorla, Marina Realidades de Emprendimiento en Pequeñas y Medianas Empresas en Chile: Estudio del Diseño como Herramienta Estratégica

estos factores en ella.

En relación con la caracterización de nivel socio económica de las comunas de Estación Central, La Florida y Ñuñoa según ingresos y educación, se tiene, tabla 1:

\section{Tabla 1}

\section{Clasificación de comunas de acuerdo con su Índice de Prioridad Social (IPS), 2019}

\begin{tabular}{llll}
\hline Comuna & Ranking & Categoría & IPS, 2019 \\
\hline Estación Central & $26^{\circ}$ & Media Alta & $69,89 \%$ \\
La Florida & $40^{\circ}$ & Baja Prioridad & $59,28 \%$ \\
Ñuñoa & $47^{\circ}$ & Sin Prioridad Social & $37,28 \%$ \\
\hline
\end{tabular}

Nota. Tabla de elaboración propia, en base al Cuadro 1 de "Región Metropolitana de Santiago. Clasificación de sus comunas de acuerdo con su índice de prioridad social". Ministerio de Desarrollo Social y Familia (2019), Seremi de Desarrollo Social, Región Metropolitana de Santiago, 2019.

Las tabla 2 y 3 desglosan cifras dadas por el IPS de las dimensiones de ingresos y educación, que son los factores principales que influyen sobre la presente investigación.

\section{Tabla 2}

\section{Indicadores de dimensión de ingresos, por comunas}

\begin{tabular}{|c|c|c|}
\hline Comuna & $\begin{array}{l}\text { Ingreso promedio imponible de } \\
\text { los afiliados vigentes al Seguro de } \\
\text { Cesantía, } 2017\end{array}$ & $\begin{array}{l}\text { Población comunal perteneciente } \\
\text { al } 40 \% \text { de menores ingresos según } \\
\text { Calificación Socioeconómica (CSE), } \\
2018\end{array}$ \\
\hline Estación Central & $\$ 1.166$ USD & $49,5 \%$ \\
\hline La Florida & $\$ 1.423$ USD & $48,3 \%$ \\
\hline Ñuñoa & $\$ 2.161$ USD & $36,4 \%$ \\
\hline
\end{tabular}

Nota. Tabla de elaboración propia, en base al Cuadro A.1 de "Indicadores Dimensión Ingreso". Ministerio de Desarrollo Social y Familia (2019), Seremi de Desarrollo Social, Región Metropolitana de Santiago. Valor dólar a diciembre de 2017.

En torno a la caracterización de las comunas de Estación Central, La Florida y Ñuñoa, los resultados dispuestos en tablas elaboradas en base al Índice de Prioridad Social establecen que Estación Central se encuentra en una posición de prioridad Media Alta; La Florida se ubica en una categoría de Baja prioridad social; y, Ñuñoa no registra ser tener prioridad social. De igual manera, esto se traduce en niveles ascendentes de ingresos económicos, reflejados en el porcentaje de la población que pertenece al $40 \%$ de menores ingresos según Calificación Socioeconómica (CSE) y el ingreso promedio imponible al seguro de Cesantía. 


\begin{tabular}{|c|c|c|}
\hline Comuna & $\begin{array}{l}\text { Porcentaje de reprobación de } \\
\text { Enseñanza Media promedio } \\
(2013-2017)\end{array}$ & $\begin{array}{l}\text { Promedio Puntaje PSU } \\
\text { (2019) DEMRE, Universidad } \\
\text { de Chile }\end{array}$ \\
\hline Estación Central & $6,6 \%$ & 479,0 \\
\hline La Florida & $6,1 \%$ & 503,6 \\
\hline Ñuñoa & $8,2 \%$ & 561,6 \\
\hline
\end{tabular}

Nota. Tabla de elaboración propia, en base al Cuadro A.3 de "Indicadores Dimensión Educación". Ministerio de Desarrollo Social y Familia (2019), Seremi de Desarrollo Social, Región Metropolitana de Santiago, 2019.

En cuanto a la dimensión de la Educación, es interesante notar que las comunas no caen en un orden ascendente tan claro, como en la dimensión de Ingresos. En cuanto al porcentaje de reprobación de la Enseñanza Media promedio, entre el año 2013 y 2017 , Nuñoa tiene el mayor índice de reprobación, mientras que La Florida se ubica en último lugar, y la comuna de menores indicadores de ingresos económicos está entre ambos lugares. Por otra parte, el promedio del puntaje de la Prueba de Selección Universitaria (PSU), del año 2019, delata que el mayor puntaje pertenece a Ñuñoa, el segundo puntaje más alto se obtiene en La Florida y el más bajo ocurre en Estación Central. Esto denota que, aunque hay mayor nivel de reprobación en Ñuñoa, aquellos estudiantes que completan la Enseñanza Media y pueden acceder a rendir la PSU, logran un puntaje mayor que el promedio de estudiantes en La Florida y Estación Central. Esta observación se puede tomar como el punto de partida para las entrevistas que vienen a continuación, y aluden a que, los recursos económicos de las personas en cada comuna, aunque denotan un factor determinante en cuánto a las dificultades para ser exitoso, son solo un aspecto del capital para emprender.

\section{Caracterización de los CDN: Entrevistas con sus Directores}

En las entrevistas que se han realizado a los expertos de los CDN han surgido cinco temas principales que se han organizado en gráficos de radar, según categorías, para establecer comparaciones y contrastes. Cabe notar que esta entrevista es parte de una investigación mayor. El gráfico 1 ha sido elaborado en base a las respuestas de los expertos a la pauta realizada por el investigador. En él, el perfil del emprendedor se configura en base a cinco categorías: edad; nivel de estudios; nivel de desarrollo de producto o servicio; necesidad de sustento; y necesidad de innovación. Estas últimas categorias responden al motivo del emprendimiento, si es necesario para mantener una familia económicamente o si hay un deseo por innovar con el emprendimiento que no está condicionado urgentemente por una necesidad financiera. La escala de valor de cada categoría varía de 1 a 50 , siendo 1 un valor nulo o inexistente, mientras que 50 implica que la categoría se cumple en su expresión máxima. 


\section{Gráfico 1 \\ Perfil de emprendedores en el CDN}

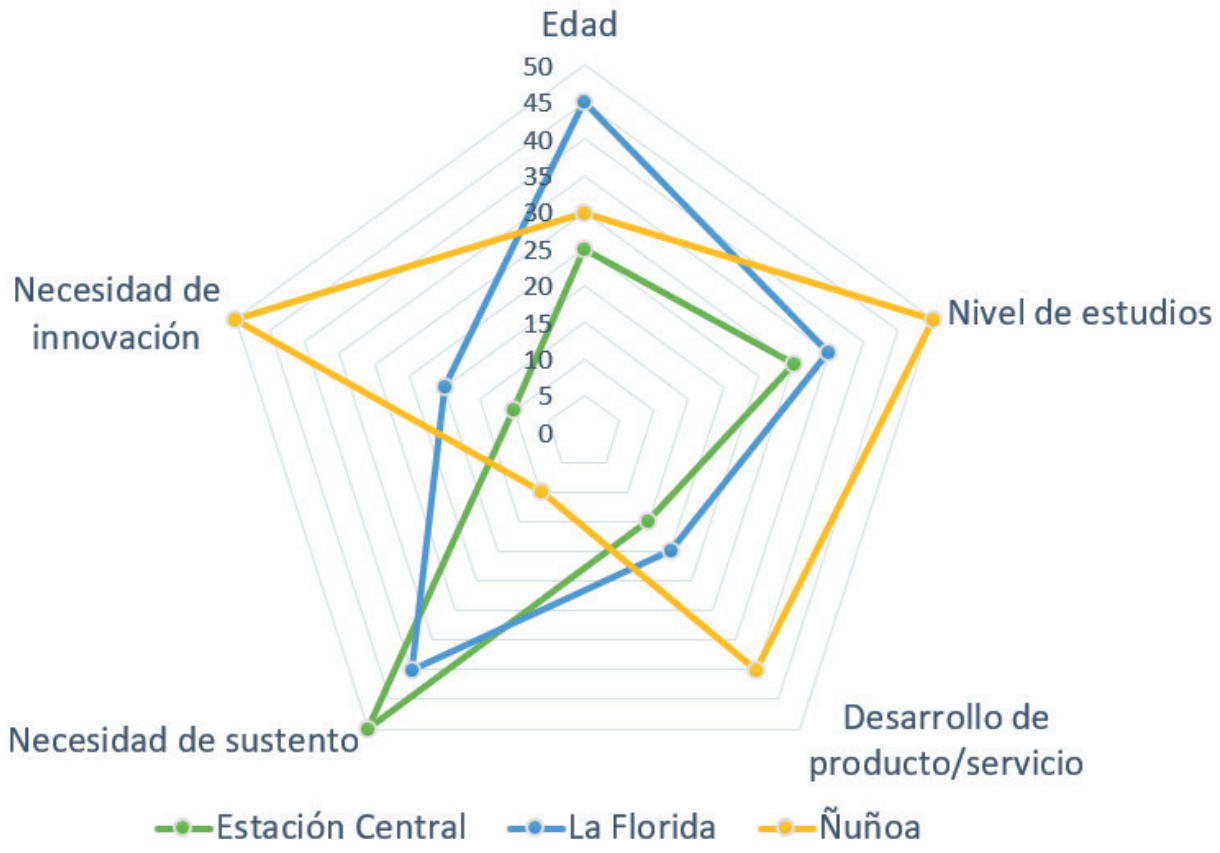

Fuente: elaboración propia

En el gráfico 2, se puede apreciar que la contabilidad tributaria es una gran urgencia para los emprendedores principalmente de Estación Central y, en un grado ligeramente menor, de La Florida. En segundo lugar, la propuesta de valor es un factor de preocupación para La Florida, Ñuñoa y, en menor grado para Estación Central. Por último, el modelo de desarrollo técnico, ubicado en un escalón superior en cuanto a estrategia de empresa, solo es considerado importante en el CDN de Ñuñoa; lo cual implica que el Diseño, presente solo en las propuestas de valor de productos y diseño de gestión en los modelos de desarrollo técnico, no es considerado una prioridad para la mayoría de los emprendedores considerados en este estudio. 


\section{Gráfico 2 \\ Urgencias del emprendedor en el CDN}

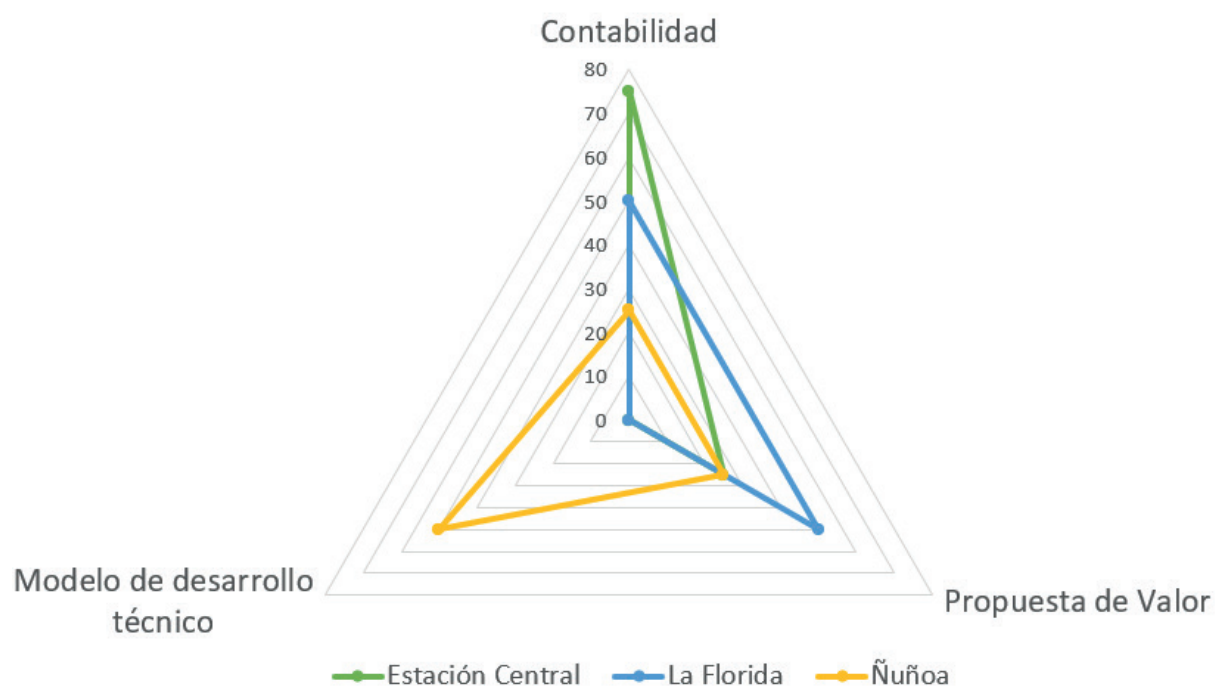

Fuente: elaboración propia

El gráfico 2 ha sido elaborado en base a las respuestas de los expertos a la pauta realizada por el investigador. En él, las urgencias del emprendedor se configuran en base a tres necesidades específicas: Contabilidad tributaria; Propuesta de Valor; y Modelo de desarrollo técnico. La escala de valor de cada categoría varía de 1 a 100 , siendo 1 un valor nulo o inexistente, mientras que 100 implica que la categoría se cumple en su expresión máxima.

En el gráfico 3, se pueden apreciar las urgencias de cada CDN, según sus clientes. La contabilidad tributaria es la principal urgencia para los emprendedores de Estación Central; la segunda urgencia en el CDN de
La Florida y de prioridad menor para Ñuñoa. Entre las prioridades, también destaca la propuesta de valor como el factor de mayor preocupación para La Florida; es el factor de segunda urgencia para Ñuñoa y, en menor grado, para Estación Central. Por último, el modelo de desarrollo técnico, ubicado en un escalón superior en cuanto a estrategia de empresa, solo es considerado importante en el CDN de Ñuñoa; lo cual implica que el Diseño, presente solo en las propuestas de valor de productos y diseño de gestión en los modelos de desarrollo técnico, no es considerado una prioridad para la mayoría de los emprendedores considerados en este estudio. 


\section{Gráfico 3 \\ La función del Diseño en el CDN}

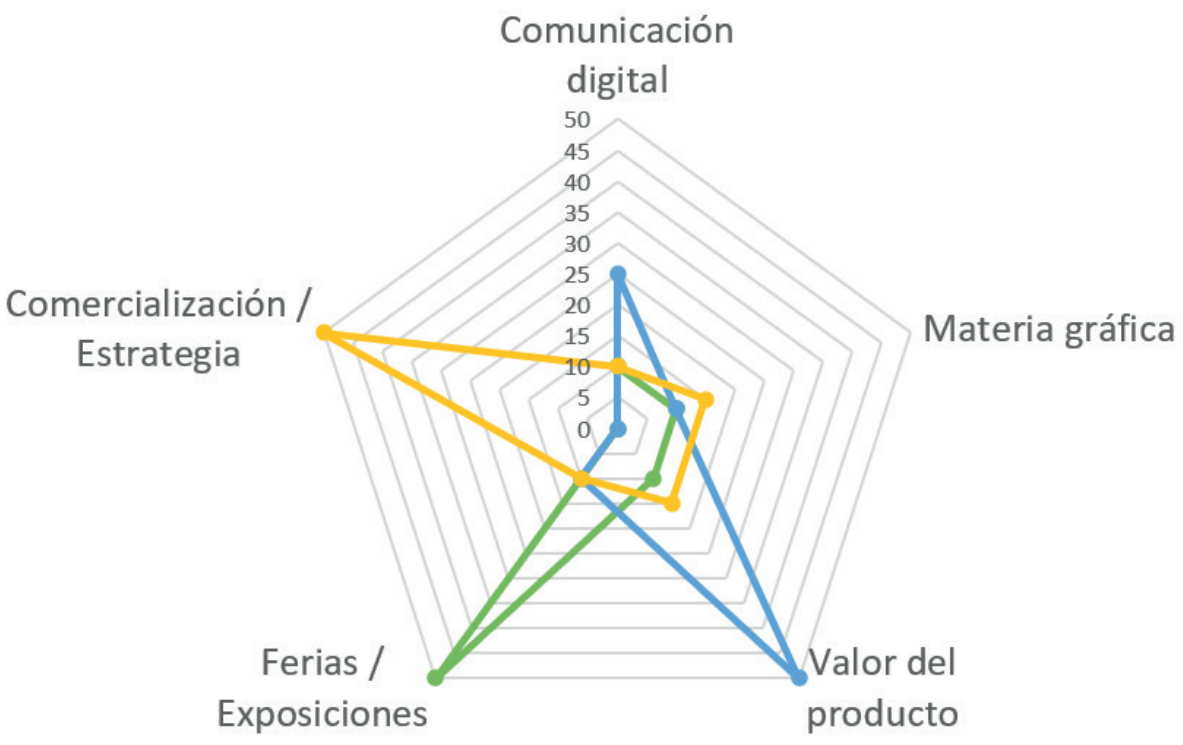

$\multimap$ Estación Central $\longrightarrow$ La Florida $\longrightarrow$ Ñuñoa

Fuente. Elaboración propia

El gráfico 3 ha sido elaborado en base a las respuestas de los expertos a la pauta realizada por el investigador. En él, la función del Diseño y su lugar en CDN está configurado por los siguientes factores: comunicación digital; materia gráfica; valor del producto; ferias I exposiciones; comercialización y estrategia. La escala de valor, en esta determinación contempla un universo de $100 \%$ de la función del Diseño, que las comunas invierten en cada categoría. Esto significa que cada comuna determina cuánto porcentaje del universo invierte en cada aspecto del Diseño, siendo $10 \%$ un valor bajo y $100 \%$ el valor completo.
El gráfico 4 indica que la mayor función que el CDN de Estación Central le atribuye al Diseño está destinada a eventos específicos como ferias $y$ exposiciones, ya que hay materias de mayor urgencia que deben ser atendidas con prioridad. A su vez, el CDN de La Florida distribuye de mejor manera su uso del Diseño, aunque se enfoca primordialmente en el valor del producto, ocupándose de elementos como la imagen y la comunicación digital. Por otra parte, el CDN de Ñuñoa pone casi toda la función del Diseño al servicio de la comercialización y estrategia de empresa, enfocándose en el diseño de modelos de gestión y, en menor grado, 
factores como ferias/exposiciones, materia gráfica y comunicación digital, ya que sus expertos consideran que estos se desprenden inherentemente de la comercialización y estrategia.

El gráfico 4 ha sido elaborado en base a las respuestas de los expertos a la pauta realizada por el investigador. En él, la importancia que cada CDN le atribuye al Ecosistema del Emprendimiento e Innovación está determinado por las siguientes categorías: vinculación entre clientes; redes con el entorno (academia, gremios y empresas); interés por la innovación; presupuesto de sus clientes para la innovación; cultura de innovación (dada por estudios superiores).

\section{Gráfico 4 \\ Sobre Ecosistemas e Innovación}

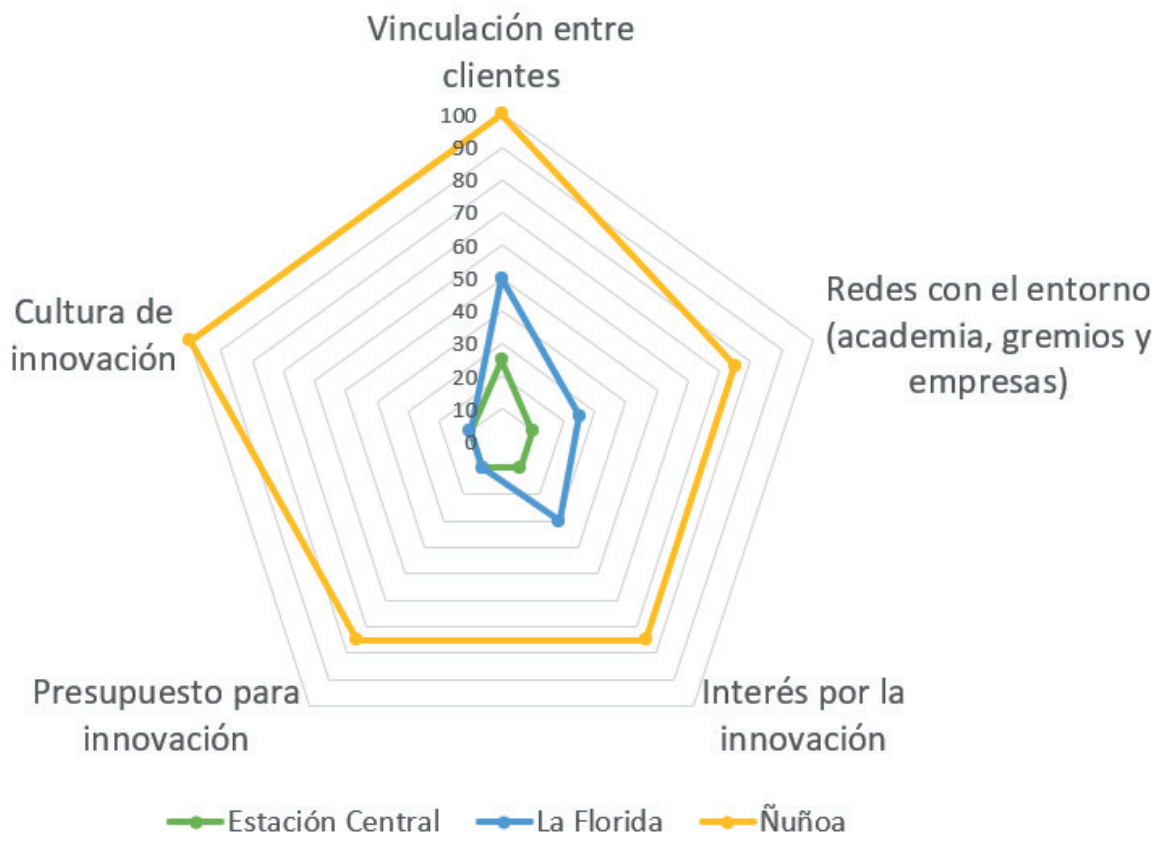

Fuente: elaboración propia 


\section{Este gráfico 4, representa} la importancia del ecosistema y la innovación en cada comuna. En cuanto al ecosistema del emprendimiento, entendido como el espacio ocupado por emprendedores de PYMEs, grandes empresas e instituciones y universidades generadoras de conocimiento, tanto Estación Central como La Florida aluden a que los clientes piden una manera de contactar fácilmente a otros emprendedores. Hacen falta redes de contacto para ubicar tanto potenciales clientes como proveedores de insumos que puedan mejorar sus ventas. Ñuñoa, por otra parte, indica que han generado redes tanto entre sus clientes como con gremios e incluso empresas públicas, pero falta un registro más formal. Además, se relacionan constantemente con instituciones generadoras de conocimiento como universidades e institutos profesionales.

En torno a la Innovación, hay grandes limitantes tanto en Estación Central como en La Florida ya que sus clientes necesitan atender urgencias financieras por sobre sus intereses de innovar. Ambos coinciden en que existe una brecha cultural entre sus clientes y la innovación, como un concepto cultural abstracto y, por ende, ajeno a ellos. Ñuñoa, en contraste, tiene mayor oportunidad para acercar la innovación a sus prácticas cotidianas ya que sus clientes tienen menor urgencia financiera. En sintonía con ello, sus asesores se especializan en la innovación como el conjunto de acciones que hacen uso del conocimiento existente para crear cosas nuevas que agreguen valor a sus participantes. Hay una teoría detrás de estas acciones en la cual otros CDNs no pueden invertir su tiempo.

\section{Perspectivas Adicionales: Datos No Cuantificables}

Además de la información que se puede graficar cuantitativamente, las cuatro categorías se pueden con otros datos cualitativos que surgen de las mismas entrevistas, con lo cual podemos esclarecer comparaciones con el IPS de cada comuna para generar vínculos entre el desempeño y el enfoque de cada CDN y el nivel socioeconómico de su comuna.

En Estación Central, llegan clientes muy heterogéneos porque esta comuna en particular es muy comercial y atienden emprendedores que trabaja en ella, pero vive en otras comunas de mayores ingresos socio económicos. Por ello, su director afirma que no hay un perfil fijo, aunque la mayoría de sus clientes suelen tener muchos problemas de orden contable y tributario. Por otra parte, en La Florida, aunque el CDN reconoce que sus clientes también tienen problemas de orden comercial, su mayor problema tiene que ver con una falta de visión. La experta dice que el enfoque principal es la propuesta de valor, desde el Diseño, porque ella tiene una relación cercana al mundo gráfico.

Esto denota que, aunque el modelo de los CDN se establece desde SERCOTEC, el enfoque de cada centro es determinado por el equipo de asesores, quienes responden a las necesidades que presentan sus clientes. De esta manera, mientras en el CDN de Estación Central se concentra más en el aspecto financiero; en La Florida, se busca invertir en recursos para la comunicación de la imagen y la propuesta de valor; y, en Ñuñoa, se trabaja la innovación como un modelo de estrategia, desde la base de los negocios que llegan. 
En este punto, podemos inferir que, Estación Central, por ser una comuna tan comercial, puede generar recursos económicos a través de sus ventas, pero no tiene el conocimiento para regular fácilmente sus obligaciones tributarias. A ello se suma una falta de saberes profesionales que limitan su concepción ante el Diseño en cuanto a imagen, comunicación y propuesta de valor. Ello explicaría el poco uso de Diseño, con la excepción de ciertos eventos que necesitan de creación gráfica, y también, de un equipo de diseñadores.

En cuanto a la innovación, los resultados de las entrevistas dejan entrever una incomprensión del concepto, el cual es erróneamente asociado principalmente a la innovación tecnológica de punta, lo cual, consecuentemente, implica un alejamiento de su utilidad práctica respecto a la realidad de los clientes de los centros. Como ejemplo, en las comunas de Estación Central y la Florida declaran dificultades para su aplicación desconociendo como acercar la innovación al día a día de los emprendedores. Una excepción a este hallazgo aparece precisamente en el centro con mejores indicadores socioculturales, Ñuñoa, en donde el encargado tiene preparación específica en el ámbito de la innovación y comprende el potencial impacto de la innovación en PYMEs y de los liderazgos que la promueven.

\section{Configuración Actual de la Realidad del Emprendimiento}

Con el conocimiento anterior, es posible revisitar el Escalamiento e Inserción del Diseño que se vio en el marco teórico para analizar de qué forma cada CDN responde a ella.

En el diagrama 1 se puede apreciar que el CDN de Estación Central se encuentra en la etapa $1 \mathrm{del}$ Escalamiento de Diseño ya que sus clientes serían empresas que no usan el Diseño en su día a día. Por otra parte, el CDN de La Florida está ubicado en el segundo escalón, dado su enfoque gráfico en el cual los clientes acceden a una mayor variedad de herramientas orientadas a la propuesta de valor en torno a imagen e identidad del producto o servicio. Por último, el CDN de Ñuñoa le otorga una importancia aún mayor al Diseño, canalizando esfuerzos tanto hacia la imagen como al desarrollo estratégico del Diseño en procesos de desarrollo de producto, difusión en redes sociales y comercialización, ubicándose entre la etapa 3 y 4 . De esta forma, al comparar el uso del Diseño del CDN de cada comuna, el diagrama 1 confirma que, a mayor uso del Diseño, mayor impacto económico y mayor éxito tiene la empresa. 


\section{Diagrama 1 \\ Escalamiento e Inserción del Diseño en empresas: EI CDN de Estación Central, La Florida y Ñuñoa en la Escalera del Diseño}

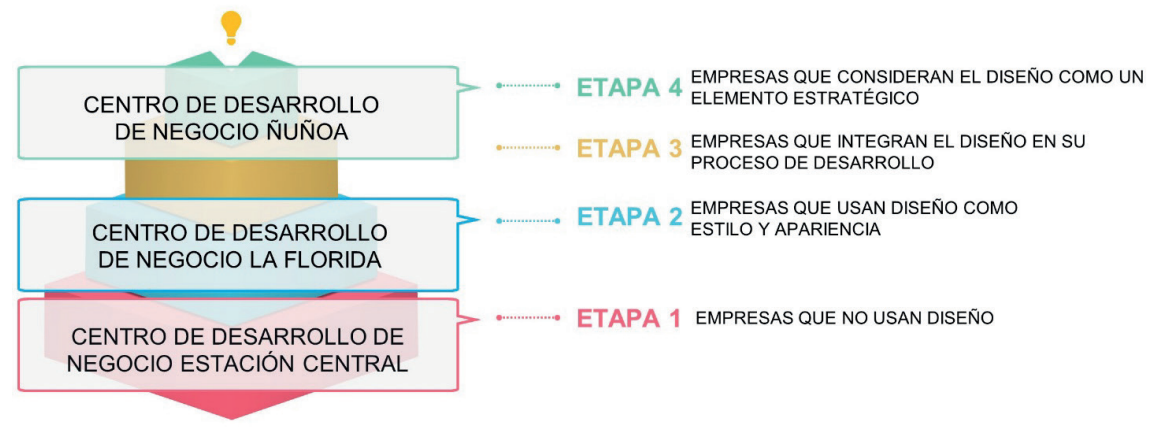

Fuente: elaboración propia

En este sentido, al integrar
el Diseño, como herramienta estratégica, dentro de los procesos del emprendimiento, esta disciplina cobra fuerza como un instrumento articulador entre todas las partes de la empresa. Esto supone que los problemas que presentan ciertos negocios, como por ejemplo de orden tributario, no se resuelven al responder específicamente a los conflictos de esa índole, sino que es importante revisar todas las áreas de la empresa en conjunto. Esto, a su vez, vuelve necesario entender la empresa como un organismo interdependiente, en el cual cada elemento tiene un impacto directo en otras áreas y no pueden tratarse como piezas autónomas. De esta idea se desprende que diseñar un plan de negocios, estratégicamente, identificando fortalezas y debilidades, y postulando metas, facilita la identificación de los pasos a seguir y los problemas que necesitan mayor atención. Este ordenamiento interdisciplinario e integrador, requiere de un cambio en el pensamiento lineal al cual los emprendedores que están iniciando sus proyectos, suelen tener.

Por ello, el CDN surge como una institución de gran ayuda para visualizar y dirigir los pasos a seguir para empezar un nuevo emprendimiento, ordenar un emprendimiento que ya está en pie, o tomar decisiones en un emprendimiento ya formalizado, dado que el Centro ofrece asesores en los distintos ámbitos que sus clientes requieran. Cabe notar, también, que una idea recurrente en el discurso de los directores de los CDN entrevistados, se remite a la soledad del micro y pequeño empresario dado por su falta de redes, por lo cual, es de gran ayuda, tanto económica y estratégica como emocional, poder contar con una variedad de asesores que lo pueden acompañar a lo largo de su emprendimiento.

\section{Conclusiones}

A partir de este estudio sobre las 
realidades de emprendimiento en $P Y M E s$ chilenas y su relación con el Diseño como una herramienta estratégica, se pueden desprender dos conclusiones claves para futuros análisis.

Los recursos tanto económicos como culturales, a modo de nivel de estudio y conocimiento, son factores determinantes en el enfoque que posee un cliente ante sus emprendimientos ya que el perfil del emprendedor está configurado por sus recursos y de ellos depende el nivel de riesgo que está dispuesto a tomar. De acuerdo con los expertos, emprender requiere no solo de un buen producto o servicio, sino de la propuesta de valor detrás, la necesidad que busca satisfacer y la estrategia para ofrecer una solución. Planificar es el primer paso hacia un emprendimiento exitoso; sin embargo, se requiere de tiempo que la urgencia económica no siempre permite.

Cabe destacar que las políticas públicas actuales, que permiten instituciones semi públicas tales como los CDN de SERCOTEC, responden a las necesidades específicas del grupo de clientes que lo conforman y que, a su vez, estas necesidades son atendidas según perspectivas $y$ prioridades específicas determinadas por los directores, asesores y equipo de trabajo de un Centro en particular. La gran falencia que se presenta de parte de los directores y de los clientes por quienes ellos hablan, se relaciona con la comunicación entre Centros y las redes de apoyo para fortalecer el ecosistema del emprendedor. El micro y pequeño emprendedor necesita de conexiones entre otros emprendedores para reforzar sus canales de comunicación con proveedores, transporte e infraestructura en general. Esto se vuelve más accesible al ser cliente del CDN e interactuar con otros emprendedores. Sin embargo, no existe un catálogo de emprendedores $\mathrm{u}$ otro tipo de registro que facilite el encuentro de clientes entre ellos. Esta es una debilidad que todos los expertos han notado y han intentado sobrellevar a través de muchas instancias de encuentros entre emprendedores.

Por otra parte, hay una falta de redes de apoyo desde grandes empresas y desde instituciones que generan conocimiento, como universidades e institutos, que puedan aportar con recursos de diversas índoles al pequeño y mediano empresario. Este último punto tiene que ver, según la experta de Estación Central, con una falta de confianza en esta área de la fuerza laboral. Sin embargo, si se recuerda que la PYME compone gran parte de la fuerza laboral nacional, podría ser necesaria una campaña dirigida específicamente a la gran industria y a la academia, en favor de la PYME. Cabe notar, además, que Chile cuenta con diversos fondos, desde las políticas públicas, que ofrecen recursos económicos para potenciar el impacto de los emprendimientos. Lamentablemente, como también notan los expertos, estos recursos son escasos ya que apuntan a empresas que ya tienen cierto grado de formalización y que, por su cantidad de requisitos, intimidan al pequeño $y$ mediano empresario.

Es pertinente advertir que, si bien los CDNs ofrecen gran ayuda, no existe una sistematización de asesorías entre ellos y cada uno responde a las necesidades que sus directores $y$ asesores perciben y dirigen según los enfoques de su administración individual. Esto implica, innegablemente, que cada Centro desarrolla una especialidad en cuanto a las capacidades que fomentan; por lo cual, el CDN Estación Central se 
Moreno Muñoz, Cristóbal; Johnson Vera, Vader; Puyuelo Cazorla, Marina

Realidades de Emprendimiento en Pequeñas y Medianas Empresas en Chile: Estudio del Diseño como Herramienta Estratégica

conoce por ser exitoso en arreglar temas de ordenamiento operativo y tributario; mientras que el CDN de la Florida se enfoca fuertemente en temas de materia gráfica y diseño; y, por último, el CDN de Ñuñoa está abocado al desarrollo estratégico de los emprendimientos, con un enfoque específico en el diseño y la innovación. Estos enfoques particulares tienen directa relación con los recursos socioeconómicos y educacionales de los clientes que acuden a cada Centro, concluyendo que la visión en torno al Diseño, y su subsecuente inversión en riesgos dirigidos a la innovación, afectan directamente en el impacto económico de sus emprendimientos.

Con estos datos, se puede concluir que existe una oportunidad en el contexto de las PYMEs para introducir la disciplina del Diseño como una herramienta estratégica que permita fortalecer el ecosistema de emprendimiento, dándoles a los emprendedores instrumentos que les permitan sofisticar sus negocios.

\section{Referencia bibliográfica}

Adriasola, F. (2019). Escenarios futuros, un complemento para métodos de innovación en Chile y Latinoamérica. RChD: creación y pensamiento, 4(7). https://rchd. uchile.cl/index.php/RChDCP/article/ view/53253

America's Small Business Development Centers. (2020, marzo). About Us. https://americassbdc.org/about-us/

Carayannis, E. G., \& Wang, V. W. L. (2012). Competitiveness model-a double diamond [Modelo de Competitividad - un diamante doble]. Journal of the Knowledge Economy, 3(3), 280-293. https:// link.springer.com/article/10.1007/ s13132-011-0038-7?shared-articlerenderer

Consejo Nacional de la Cultura y las Artes. (2017). Política de Fomento del Diseño (2017 - 2022). Santiago: Ministerio de las Culturas, las Artes y el Patrimonio. https://bit.ly/3raOSme

Fleetwood, R. (2005). Design audit by research: Building a knowledge base for competitiveness by design. Joining forces, 1-8.

Fonseca, A. C. (2008). Economía creativa como estrategia de desarrollo: una visión de los países en desarrollo. São Paulo: Itaú Cultural.

Gamonal, R. (2011). La disciplina del diseño desde la perspectiva de las ciencias sociales. Revista prisma social, 7, 1-19. https://pixabay.com/ es/vectors/search/?cat=science

Lawlor, P., O'Donoghue, A., Wafer, B., y Commins, E. (2015). Design Driven Innovation: Why it Matter for SME Competitiveness [Diseño impulsado por la Innovación: Porqué importa para la Competitividad en PYME]. The Circa Group Europe, Northern \& Western Regional Assembly. http://edepositireland.ie/ handle/2262/82846

Leinonen, T., \& Gazulla, E. D. (2014). Pensamiento de diseño y aprendizaje colaborativo. Design thinking and collaborative learning. Comunicar. Media Education Research Journal, 22(1). https://doi. org/10.3916/C42-2014-10

Maldonado Guzmán, G., García Ramírez, R., Mata Zamores, S., y Castillo Esparza, M. (2021). Innovación abierta, crecimiento y rendimiento en la pyme de la industria automotriz de México. Telos: revista de Estudios Interdisciplinarios en Ciencias Sociales, 23(1), 85-99. www.doi. 


\section{org/10.36390/telos231.07}

Ministerio de Desarrollo Social y Familia. (2019). Región Metropolitana de Santiago Índice de Prioridad Social de Comunas 2019 Seremi de Desarrollo Social y Familia Metropolitana. Recuperado de https://bit.ly/2TdHvOF

Ministerio de Economía, Fomento y Turismo. (2014). Antecedentes para la revisión de los criterios de clasificación del Estatuto Pyme. Recuperado de https://bit. Iy/2UTHmiG

Moreno, C. \& Jeno, F. (2018). Taller de diseño industrial: una aproximación al modelo metodológico proyectual, Universidad De Santiago De Chile. Revista de Pedagogía, 39(105). http://saber. ucv.ve/ojs/index.php/rev ped/article/ view/16498

Moreno, C., Aguirre, S., \& Puyuelo, M. (2019). El diseño en las pequeñas y medianas empresas: Un estudio de caso del Centro de Desarrollo de Negocios de Estación Central en Chile. Revista Espacios, 40(43), 18.

https://www.revistaespacios.com/ a19v40n43/a19v40n43p18.pdf

Nordfors, D., Cerf, V., \& Senges, M. (2016). Disrupting Unemployment: Reflection on a Sustainable, Middle Class Economic Recovery. Ewing Marion Kauffman Foundation.

Ortega, M. S., \& Ceballos, P. B. (2015). Design thinking: Lidera el presente. Crea el futuro. Esic editorial. https://bit.ly/3eqzkW6

Puente, R., González, C. G., \& Cervilla, M. A. (2019). Necessity entrepreneurship in Latin America: it's not that simple. Entrepreneurship
\& Regional Development, 31(9-10), 953-983. https://doi.org/10.1080/089 85626.2019.1650294

Ramlau, U. H. (2004). In Denmark, design tops the agenda [En Dinamarca, el diseño está en la cima de la agenda]. Design Management Review, 15(4), 48-54. https://doi. org/10.1111/j.1948-7169.2004. tb00182.x

Robertt, P., \& Lisdero, P. (2016). Epistemología y metodología de la investigación sociológica: reflexiones críticas de nuestras prácticas de investigación. Sociologías, 18(41), 54-83. https://www.scielo.br/j/soc/a/ dPxhdSbtHZK5cKfHqM6MpCy/ abstract/?lang $=e s$

Servicio de Cooperación Técnica SERCOTEC. (2019). SERCOTEC. https://www.SERCOTEC.cl/quienessomos/

Viladás, X. (2010). Diseño rentable: Diez temas a debate. IndexBook. https://www.casadellibro.com/ libro-diseno-rentable-diez-temas-adebate/9788496774827/1229672

Yacuzzi, E. (2005). El estudio de caso como metodología de investigación: teoría, mecanismos causales, validación. Serie Documentos de Trabajo (No. 296). https://ucema. edu.ar/publicaciones/download/ documentos/296.pdf

Solis Muñoz, J. B., Neira Neira, M. L., Ormaza Andrade, J. E., \& Quevedo Vázquez, J. O. (2021). Emprendimiento e innovación: Dimensiones para el estudio de las MiPymes de Azogues-Ecuador: Revista De Ciencias Sociales (Ve), 27(1), 315-333. https://doi. org/10.31876/rcs.v27i1.35318 